\title{
Rapid Detection of Ractopamine and Salbutamol in Swine Urine by Immunochromatography Based on Selenium Nanoparticles
}

This article was published in the following Dove Press journal: International Journal of Nanomedicine

\author{
Zhizeng Wang $\mathbb{D}^{1, *}$ \\ Qianqwen Zhou ${ }^{1, *}$ \\ Yafei Guo ${ }^{2, *}$ \\ Hangzhan $\mathrm{Hu}^{\prime}$ \\ Zhi Zheng' \\ Shulian $\mathrm{Li}^{\mathrm{I}}$ \\ Yaohui Wang (D) \\ Yuanfang $\mathrm{Ma}^{\mathrm{I}}$
}

'Joint National Laboratory for Antibody Drug Engineering, School of Basic Medical Sciences, Henan University, Kaifeng, 475004, People's Republic of China; ${ }^{2}$ School of Laboratory, Sanquan college of Xinxiang Medical University, Xinxiang, Henan, 453003, People's Republic of

China

*These authors contributed equally to this work
Correspondence: Yaohui Wang; Yuanfang

$\mathrm{Ma}$

Joint National Laboratory for Antibody Drug Engineering, School of Basic Medical

Sciences, Henan University, Kaifeng, 475004,

People's Republic of China

Tel +86 I82378I6739; +86 I3803786I39

Email wangyaohui200I@sina.com;

mayf@henu.edu.cn
Purpose: The purpose of this study was to establish a lateral flow immunoassay using selenium nanoparticles (Se-NPs) as a probe to detect ractopamine (RAC) and salbutamol (SAL) in swine urine.

Methods: SDS and PEG were used as templates to prepare Se-NPs; anti-RAC monoclonal antibodies or anti-SAL monoclonal antibodies were labelled with Se-NPs; and rapid detection kits were prepared. The sensitivity, specificity, and stability were measured, and actual samples were analysed.

Results: The Se-NPs were spherical with a diameter of $40.63 \pm 5.91 \mathrm{~nm}$, and were conjugated successfully with an anti-RAC antibody to give a total diameter of $82.33 \pm$ $17.91 \mathrm{~nm}$. The detection limit of a RAC kit in swine urine was $1 \mathrm{ng} / \mathrm{mL}$, and that of a SAL kit was $3 \mathrm{ng} / \mathrm{mL}$. Both procedures could be completed within 5 minutes. No cross-reaction occurred with clenbuterol, bambuterol and phenylethanolamine A. Samples were tested consistently across different batches of kits for swine urine. The results of the kits were identical to those of actual clinical samples analysed by ELISA, and the coincidence rate was $100 \%$.

Conclusion: The assay kit does not require any special device for reading the results, and the readout is a simple colour change that can be evaluated with the naked eye. It is easy to operate, sensitive, specific, and stable This kit is suitable for the rapid and real-time detection of RAC and SAL residues in swine urine samples.

Clinical Trial Registration: Swine urines samples were used under approval from the Experimental Animal Ethics committee of the Joint National Laboratory for Antibody Drug Engineering, Henan University.

Keywords: ractopamine, salbutamol, lateral flow, selenium nanoparticles

\section{Introduction}

Ractopamine (RAC) and salbutamol (SAL) are $\beta$-agonists, as are several drugs collectively called "clenbuterol". 1 RAC and SAL were originally used to treat bronchitis and asthma. In recent years, they have been illegally used to increase the feed conversion rate in animal husbandry because the large-scale use of $\beta$-agonists can promote animal body fat decomposition and protein synthesis. ${ }^{2,3}$ However, residual RAC and SAL in the animal body will harm human health after entering the human body, causing such effects as dizziness, tachycardia and amyostasia, and even death. ${ }^{4}$ Therefore, the use of RAC and SAL in animal feeds has been banned in most countries. $^{5,6}$ However, RAC and SAL are still illegally used in animal production. 
For example, in 2017, SAL was found in raw pork purchased by a middle school canteen in Jinan City, and in 2018, RAC was detected in pig tongues by the food and drug law enforcement branch of Quanzhou City. Therefore, the portable detection of RAC and SAL is necessary to strengthen market monitoring.

The current detection methods of RAC and SAL are mainly divided into physical-chemical analysis and immuno-analysis. Physical-chemical analysis methods include high-performance liquid chromatography (HPLC), ${ }^{7,8}$ gas chromatography-mass spectrometry (GCMS), ${ }^{9,10}$ and liquid chromatography-mass spectrometry (LC-MS). ${ }^{11}$ These methods offer the advantages of high accuracy, good repeatability and quantitative realization. However, the instruments are expensive, and their operation is complicated and requires professional personnel; moreover, these approaches cannot meet the need for rapid, largescale sample screening and are therefore limited in practical applications. ${ }^{12}$ Immunoassays mainly include the enzymelinked immunosorbent assay (ELISA), ${ }^{13,14}$ chemiluminescence immunoassay (CLIA) ${ }^{15}$ and immunochromatography (ICA). ${ }^{16,17}$ ELISA has the advantages of high sensitivity and high throughput, but it also presents the disadvantages of a high false positive rate and inability to provide rapid onsite detection. ICA is a rapid detection technique involving colloidal gold, ${ }^{18}$ quantum dots, ${ }^{19}$ fluorescence microspheres, ${ }^{20}$ etc. It consists of a sample pad, bonding pad, reaction pad and absorbent pad (Figure 1A). Colloidal gold immunochromatography has the advantages of simple and rapid operation and is suitable for the on-site detection of large-scale samples. ${ }^{21}$ Thus, it has been widely used in the field of veterinary drug residue detection. However, detection using colloidal gold as a probe exhibits relatively low sensitivity. Moreover, high-temperature boiling is required in the relatively complicated preparation process of colloidal gold, and the raw material is relatively expensive. Compared with colloidal gold, selenium nanoparticles (Se-NPs) can be used as an immunochromatographic probe with higher sensitivity and stability as well as lower cost, and they can be prepared at room temperature. Se-NPs have the advantages of structural stability, resistance to electrolyte damage, and an economical raw material cost, and their use in the preparation of chromatographic test strips has been gradually increasing. ${ }^{22,23}$ In this study, Se-NPs were used as a marker to establish a highly sensitive and low-cost immunochromatographic method for the rapid in situ detection of RAC and SAL.

\section{Materials and Methods \\ Materials and Apparatus}

Polyethylene glycol (PEG) was purchased from the YuanYe Biological Technology Co., Ltd (Shanghai, China). Ascorbic acid (Vc) and selenous acid $\left(\mathrm{H}_{2} \mathrm{SeO}_{3}\right)$ were obtained from Xiya Reagent (Shandong, China). Bovine serum albumin (BSA) was purchased from Sigma. The corresponding RAC and SAL monoclonal

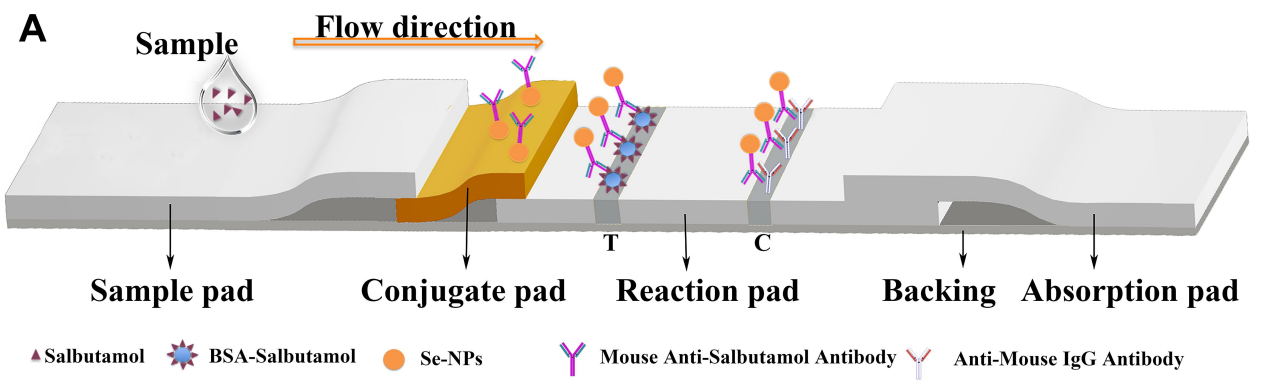

B
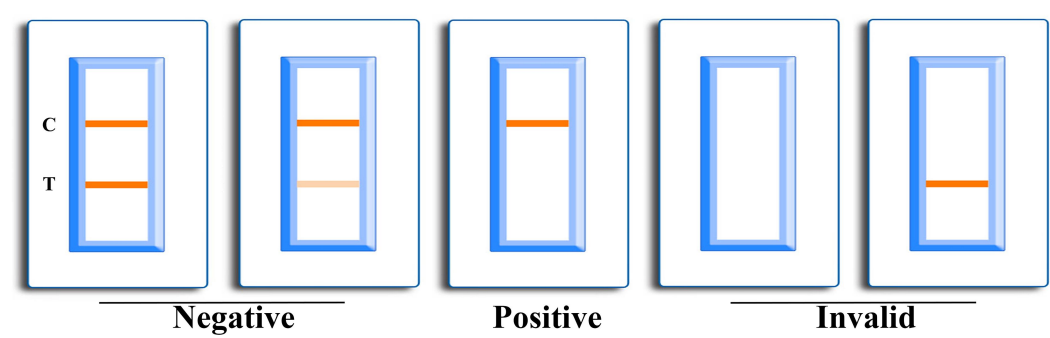

Figure I Diagram and components of the immunochromatographic test strip for ractopamine or salbutamol (A) and visual assessment guidelines for interpreting the test strip results $(\mathbf{B})$.

Abbreviations: C, control line; T, test line. 
antibodies (Abs) were acquired from the Baiaotong Experimental Material Centre (Luo Yang, China). The RAC and SAL ELISA kits were purchased from the Fen De Biotechnology Co., Ltd (Shen Zhen, China). Deionized water (Millipore Milli-Q Grade) with a resistivity of 18.2 $\mathrm{M} \Omega \cdot \mathrm{cm}$ was used throughout this work. The dispenser and sprayer XYZ 3010 and the guillotine cutter were provided by the Shanghai JieNing Biological Technology Co., Ltd (Shanghai, China). An electric blast drying oven was provided by Shanghai YiHeng Scientific Instruments Ltd (Shanghai, China).

\section{Preparation of Selenium Nanoparticles}

Se-NPs were prepared by the vitamin $\mathrm{C}$ reduction of $\mathrm{H}_{2}$ $\mathrm{SeO}_{3}$ using SDS and PEG as templates. First, $85 \mathrm{~mL}$ of ultrapure water was added to a $250 \mathrm{~mL}$ clean beaker and placed on a magnetic stirrer, and $0.5 \mathrm{~g}$ PEG was added and stirred well. Then, 0.1 M SDS solution $(5 \mathrm{~mL})$ was added and stirred well; $3.2 \mathrm{M}$ vitamin $\mathrm{C}$ solution $(0.5 \mathrm{~mL})$ was added and stirred well; and $0.4 \mathrm{M} \mathrm{H}_{2} \mathrm{SeO}_{3}$ solution $(0.5 \mathrm{~mL})$ was added. The solution gradually changed from colourless to orange, and stirring was continued for 15 minutes. After the reaction was completed, the Se-NP solution was prepared. To the naked eye, the solution was a clear and transparent orange-red liquid with no turbidity or surface floating objects. One millilitre of Se-NP solution was prepared in a clean centrifuge tube and centrifuged for 10 minutes at a rate of $9600 \mathrm{~g}$, and the prepared Se-NPs were resuspended. The stability of the Se-NP solution was verified by dynamic light scattering (DLS) ${ }^{24}$ and transmission electron microscopy (TEM). ${ }^{23}$

\section{Strip Preparation}

The dimensions of each part of the test strip are as follows: sample pad $(20 \mathrm{~mm} \diamond 4 \mathrm{~mm})$, conjugate pad $(2.8 \mathrm{~mm} \diamond$ $4 \mathrm{~mm})$, absorbant pad $(18 \mathrm{~mm} \diamond 4 \mathrm{~mm})$, NC membrane $(25 \mathrm{~mm} \diamond 4 \mathrm{~mm})$ and backing $(60 \mathrm{~mm} \diamond 4 \mathrm{~mm})$.

\section{Preparation of the Reaction Pad}

The nitrocellulose membrane (Millipore 135) was cut to $25 \mathrm{~mm}$ wide and $100 \mathrm{~mm}$ long and then pasted to the middle of the PVC substrate. Goat anti-mouse IgG (1 mg/ $\mathrm{mL})$ or goat anti-rabbit $\mathrm{IgG}(1 \mathrm{mg} / \mathrm{mL})$ was dispensed onto the control area to prepare the control line, and RAC-BSA $(1 \mathrm{mg} / \mathrm{mL})$ or SAL-BSA $(0.05 \mathrm{mg} / \mathrm{mL})$ was dispensed onto the detection area to prepare the test line. After 3 hours of drying at $37^{\circ} \mathrm{C}$ in an electric blast drying oven, the samples were removed and placed into a sealed plastic bucket.

\section{Preparation of Conjugation Pad}

Next, $\mathrm{K}_{2} \mathrm{CO}_{3}$ was used to adjust the $\mathrm{pH}$ of the Se-NP solution, anti-RAC or anti-SAL monoclonal antibody $(\mathrm{McAb})$ was added, and the solution was mixed at room temperature for 30 minutes to allow the $\mathrm{McAb}$ to conjugate fully to the Se-NPs. After labelling, BSA was added to the solution to a final concentration of $1 \%$, and the samples were mixed for 30 minutes to seal the exposed sites on the Se-NPs. After centrifugation, the supernatant was removed, and the precipitate was suspended in working solution $(\mathrm{pH}=7.410 \mathrm{mM}$ PBS containing $0.05 \%$ Tween 20, 5\% sucrose, 1\% BSA) and spread evenly on a glass fibre membrane. The conjugates of Se-NPs combined with McAbs were solidified on the glass fibre membrane after being dried for 3 hours in the electric blast drying oven at $37^{\circ} \mathrm{C}$.

\section{Preparation of the Sample Pad}

The glass fibre was soaked in the sample point solution $(\mathrm{pH}=7.410 \mathrm{mM}$ PBS containing $0.1 \%$ Tween $20,1 \%$ BSA) for 15 minutes and dried at $37^{\circ} \mathrm{C}$ for 12 hours.

\section{Selenium Nanoparticle Kit Procedure}

Determination of the RAC Se-NP rapid test kit (Figure 1B): the kit was prepared by a competitive method so that when the sample does not contain RAC, the redissolved Se-NP-anti-RAC McAb complex reacts with RACBSA of the $\mathrm{T}$ line and results in cumulative colouration. The goat anti-mouse IgG coating at the $\mathrm{C}$ line also reacts with the redissolved complex. Therefore, when the $\mathrm{C}$ line and $\mathrm{T}$ line are both coloured, the result is judged to be negative. When there is enough RAC in the sample, only the $\mathrm{C}$ line shows colour, and the $\mathrm{T}$ line does not show colour, which is a positive result. The same principle holds for the SAL kit.

\section{Determination of the Optimal $\mathrm{pH}$ and Labelled Concentration}

The labelling $\mathrm{pH}$ will affect the labelling effect of the nanoparticles and antibody, and the labelling concentration is related to the sensitivity of the kit. Different concentrations of anti-RAC McAb were labelled with Se-NPs with different $\mathrm{pH}$ gradients, and RAC-BSA and goat antimouse IgG were coated at the detection and control lines, respectively. Different concentrations of anti-SAL $\mathrm{McAb}$ were labelled with Se-NPs with different $\mathrm{pH}$ 
gradients, and SAL-BSA and goat anti-rabbit IgG were coated at the test and quality control lines, respectively. The optimal $\mathrm{pH}$ and labelled concentration were determined according to the colour of the test line in both negative and positive samples. For $\mathrm{pH}$ adjustment, we set a value every $0.5 \mathrm{pH}$ and determined the optimal $\mathrm{pH}$ through the colour presentation effect of the test line of the kit. The concentration of conjugated antibody was adjusted by setting a gradient every $2 \mu \mathrm{g} / \mathrm{mL}$, and the optimal conjugating concentration was determined through the colour presentation effect of the test line of the kit.

\section{Sensitivity, Specificity, Stability, Recovery Experiment and Clinical Sample Verification of the Selenium Nanoparticle Kits}

We placed $80 \mu \mathrm{L}$ of standard sample diluted with negative swine urine on a kit, observed the change in the $\mathrm{C}$ line and $\mathrm{T}$ line in 5 minutes, and determined that the concentration of the sample was at the detection limit when the $\mathrm{T}$ line was colourless. In addition, clenbuterol hydrochloride, salbutamol hydrochloride and ractopamine hydrochloride were added to test the kit specificity. Samples from the same batch of Se-NP test strips were stored in an electronic moisture-proof cabinet at $40{ }^{\circ} \mathrm{C}$ and with $30 \%$ humidity for 30 days to verify their stability. All the detected kits were analysed by ImageJ, and the results were visualized with GraphPad Prism.

Different concentrations of RAC and SAL were detected for the recovery experiment with the corresponding ELISA kit. Each concentration test was repeated not less than three times, with the SD value and CV\% calculated by SPSS.

We randomly collected 16 urine samples from a pig farm and tested them with the Se-NP kit. ELISA was used to determine the samples in parallel to compare the coincidence rate of the results of the two different methods.

\section{Results}

\section{Characterization of Selenium} Nanoparticles and Se-NPs Conjugated with Antibody

The Se-NP solution was prepared by using ascorbic acid to reduce $\mathrm{H}_{2} \mathrm{SeO}_{3}$ with SDS and PEG as stabilizers. The orange solution was clear and bright, with no suspended impurities on the surface and no agglomerated particles at the bottom of the solution. Se-NPs were conjugated with anti-SAL or anti-RAC McAb and centrifuged, and the precipitate from the solution was dispersed in the bottom of the centrifuge tube and could be collected. Simultaneously, a Tyndall effect was observed, as shown in Figure 2A. The hydrated particle size was $69.37 \mathrm{~nm}$, with a 0.069 polydispersity index (PDI) value and -22.8 $\mathrm{mV}$ zeta potential. The particle sizes of Se-NPs labelled with anti-SAL McAb and anti-RAC McAb are $78.03 \mathrm{~nm}$ and $86.11 \mathrm{~nm}$, respectively. Therefore, Se-NPs were successfully coupled with anti-SAL McAb and anti$\mathrm{RAC} \mathrm{McAb}$, and their performance is stable, as indicated by the PDI value detected by the DLS (Figure 2B). The Se-NPs had a spherical structure with a diameter of $40.63 \pm 5.91 \mathrm{~nm}$ (Figure 2C and $\mathrm{E}$ ), and successful coupling with anti-RAC antibody resulted in a size of $82.33 \pm 17.91 \mathrm{~nm}$ according to TEM (Figure $2 \mathrm{D}$ and $\mathrm{E})$.

\section{Optimization of the Selenium Nanoparticle Kits}

$\mathrm{pH}$ can affect the binding of Se-NPs and protein. In this experiment, an orange line appeared at the control line in $4 \mathrm{pH}$ gradients, which indicated that the Se-NPs and anti-RAC McAb were successfully observed. It was found that $\mathrm{pH} 8.0$ was the best for both colour development and sensitivity. When the $\mathrm{pH}$ during protein labelling was 6.0 or 7.0 , the conjugate was not effective resuspended after centrifugation, and the Se-NP kits did not show the test line for negative samples. The colour rendering effect in the test line or control line at $\mathrm{pH} 7.5$ and $\mathrm{pH} 9.0$ was worse than at $\mathrm{pH}$ 8.0. Therefore, a $\mathrm{pH}$ of 8.0 is the optimal $\mathrm{pH}$ for the RAC Se-NP kit (Figure $3 \mathrm{~A}$ and $\mathrm{B}$ ). The SAL Se-NP kit was determined to have an optical $\mathrm{pH}$ of 8.0 by the same method (Supplement Figure $1 \mathrm{~A}$ and $\underline{\mathrm{B}}$ ).

After adjusting the $\mathrm{pH}$ of the Se-NPs solution to 8.0, we labelled it with different concentrations of anti-RAC $\mathrm{McAb}$. It was found that the $\mathrm{C}$ line appeared but that the $\mathrm{T}$ line did not appear for the detection of negative samples when the labelled anti-RAC McAb content was $4.0 \mu \mathrm{g} / \mathrm{mL}$. When the labelled protein concentration was $8.0 \mu \mathrm{g} / \mathrm{mL}$, the $\mathrm{C}$ and $\mathrm{T}$ lines were faint, and the stability in detecting negative samples was poor. When the tagged antibody concentration was $6.0 \mu \mathrm{g} / \mathrm{mL}$, the effect of the kit was good with regard to both colour 

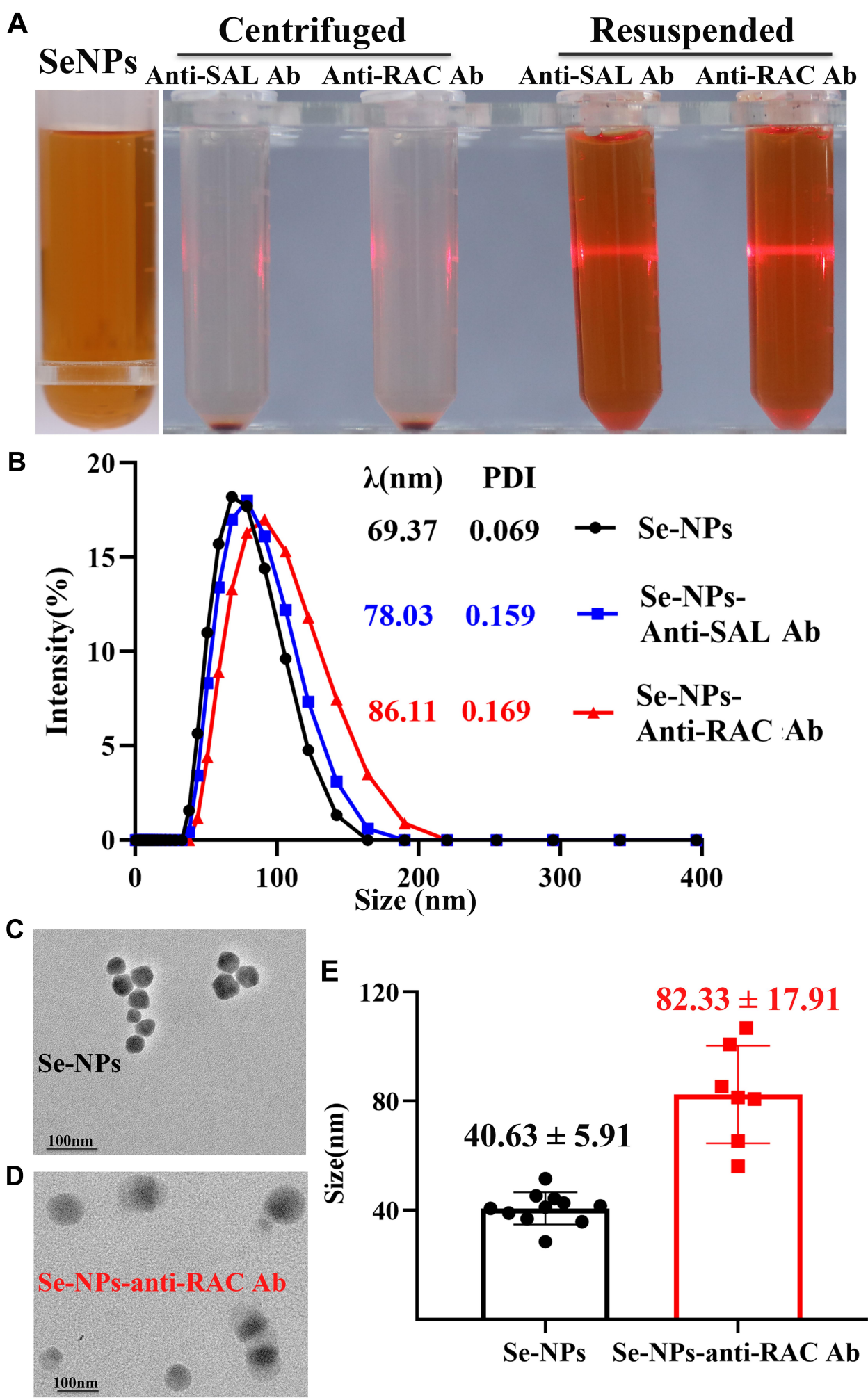

Figure 2 Characterization of Se-NPs and of Se-NPs conjugated with antibody: (A) images of Se-NPs, the complex after centrifugation, and the complex after suspension. (B) DLS results. (C) TEM result of Se-NPs, and (D) conjugation with anti-RAC antibody and (E) statistical results.

Abbreviations: Se-NPs, selenium nanoparticles; DLS, dynamic light scattering; TEM, transmission electron microscopy; RAC, ractopamine; SAL, salbutamol; Ab, antibody.

development and sensitivity. Therefore, $6.0 \mu \mathrm{g} / \mathrm{mL}$ was the optimal labelling concentration for the RAC Se-NP kit (Figure 3C and D). By the same method, the optimal labelling concentration for the SAL Se-NP kit was determined to be $4.0 \mu \mathrm{g} / \mathrm{mL}$ (Supplement Figure 1C and D). 


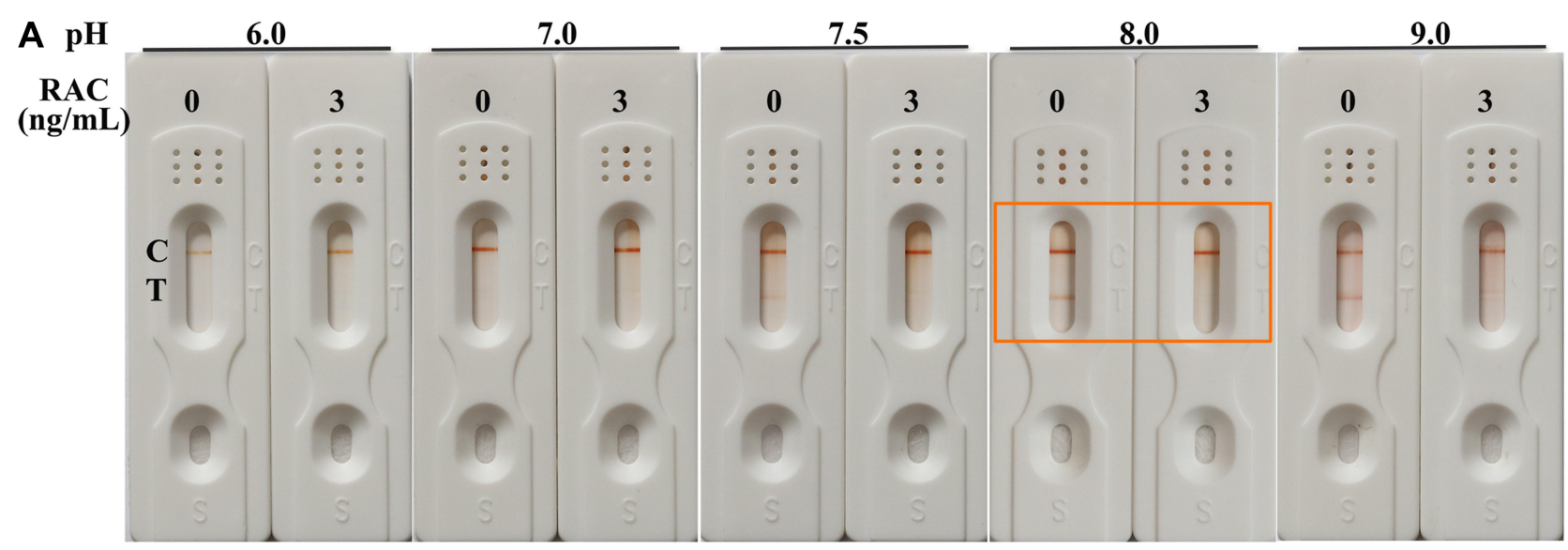

B

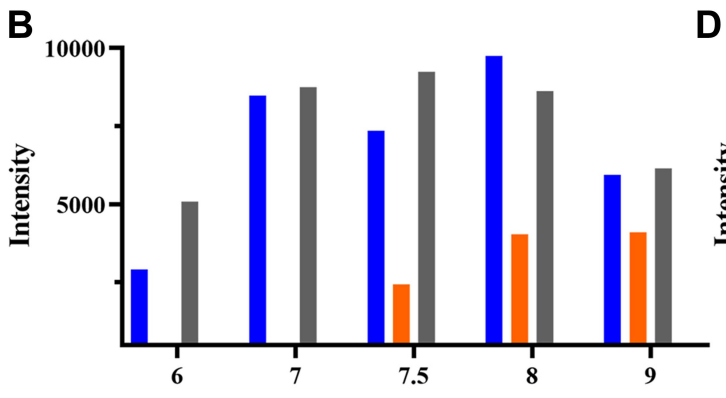

D

C

pH of Se-NPs-anti-RAC Ab

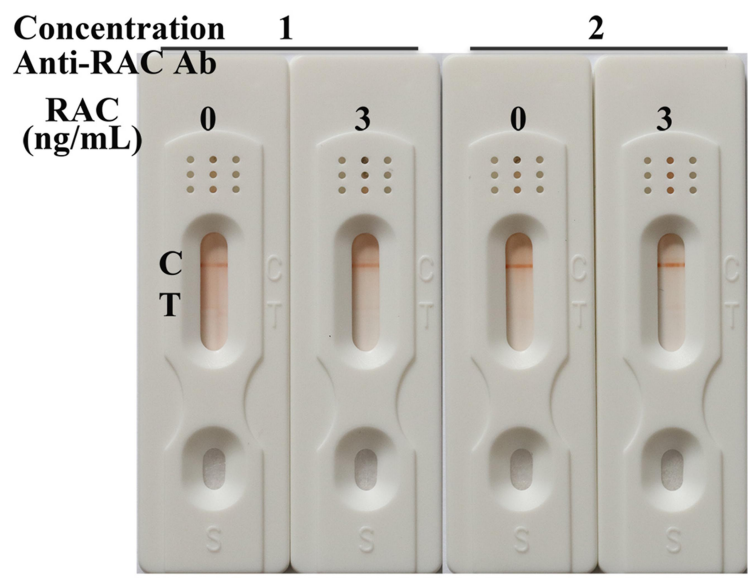

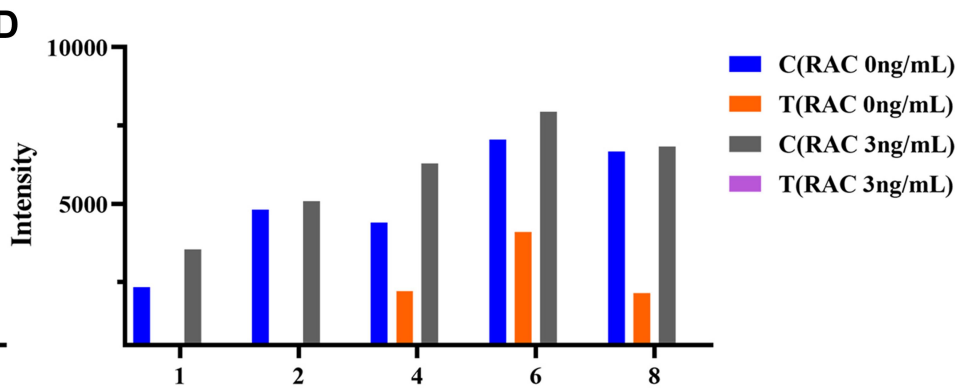

Concentration of Se-NPs-anti-RAC Ab (ng/mL)

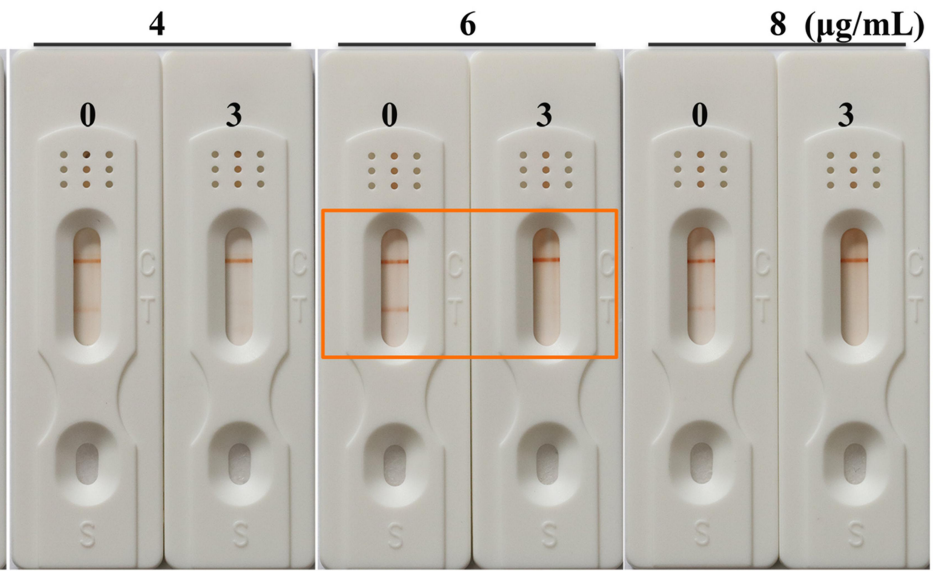

Figure 3 Optimal $\mathrm{pH}$ and labelling concentration of the ractopamine (RAC) kit. Detection results (A) and auxiliary judgement result (C) with ractopamine kit at different $\mathrm{pH}$ values; detection results (B) and auxiliary judgement result (D) with ractopamine kit at different concentrations. Orange boxes: best colour rendering results. Abbreviations: $\mathrm{C}$, control line; T, test line.

\section{Sensitivity, Specificity and Stability of the Selenium Nanoparticle Kits}

As shown in Figure 4A, the control lines of all Se-NP kits were coloured, and the results of the Se-NP kits were negative when the standard concentration of SAL was $0 \mathrm{ng} / \mathrm{mL}$ to $2 \mathrm{ng} / \mathrm{mL}$; that is, the $\mathrm{C}$ line and $\mathrm{T}$ line were both coloured. The results were positive when the SAL standard concentration was $3 \mathrm{ng} / \mathrm{mL}$ or $4 \mathrm{ng} / \mathrm{mL}$, and the $\mathrm{T}$ line did not appear orange. The naked eye results were the same as the results analysed by ImageJ (Figure 4B). Therefore, the sensitivity of the SAL Se-NP kit was $3 \mathrm{ng} / \mathrm{mL}$, which exceeds the market standard; the fitting curve was $\mathrm{y}=-10113 \mathrm{LgC}+5960$ with an $\mathrm{R}^{2}$ value of 0.9786 (Figure 4C). Similarly, the sensitivity of the RAC Se-NP kit was $1 \mathrm{ng} / \mathrm{mL}$ by naked eyes (Figure 4D) and ImageJ (Figure 4E), and $y=-1745.2 \mathrm{LgC}+876.56$ with an $\mathrm{R}^{2}$ value of 0.9503 (Figure $4 \mathrm{~F}$ ). Compared with the works of $\mathrm{Li}$ et $\mathrm{al}^{25}$ and others using colloidal gold 


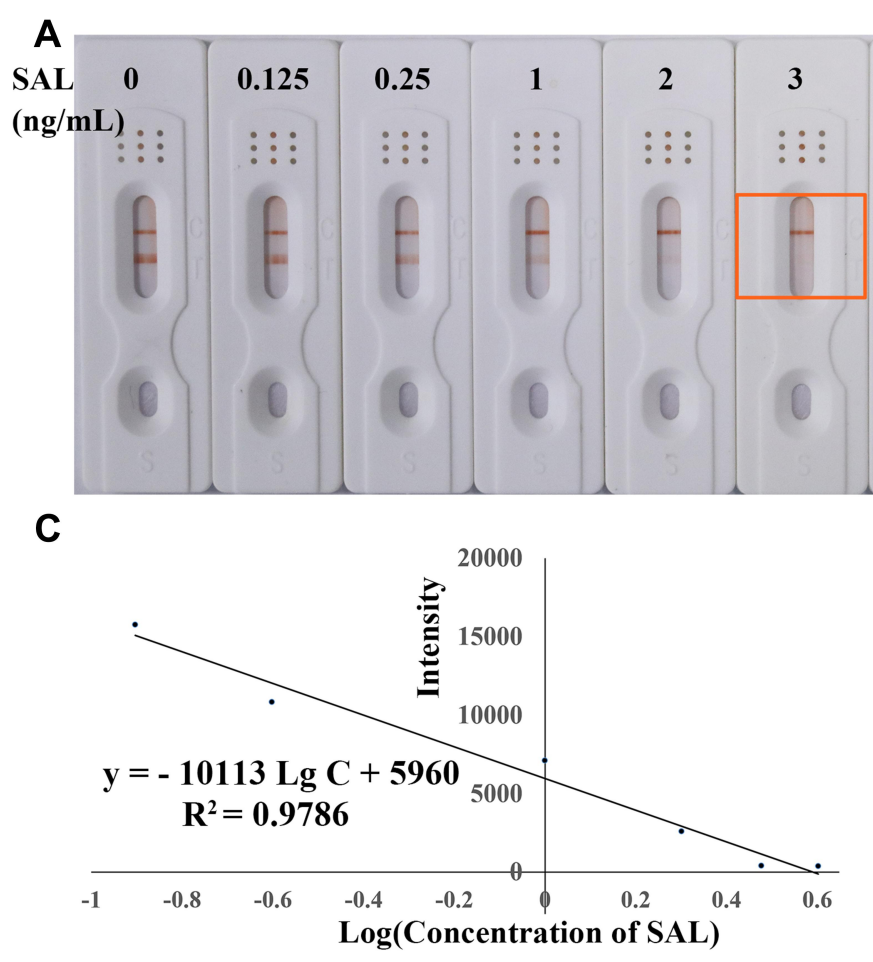

D

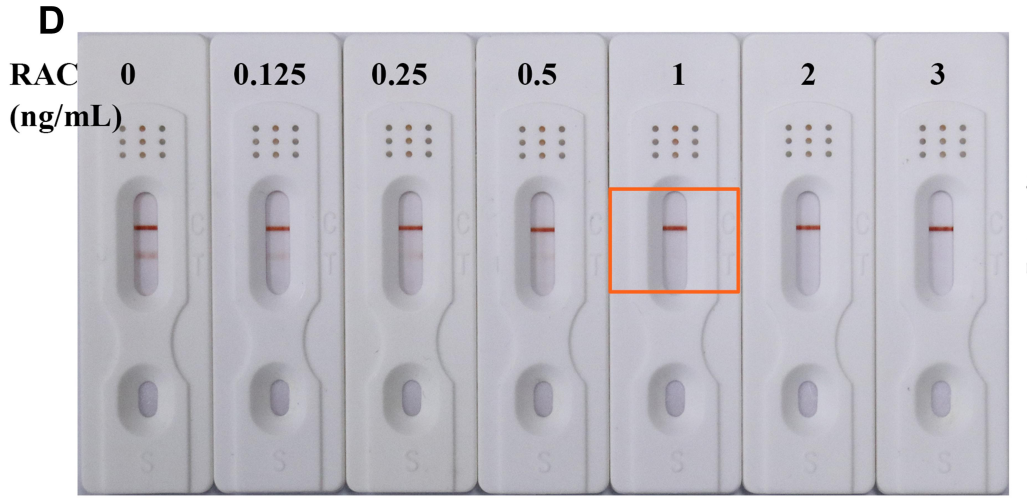

B

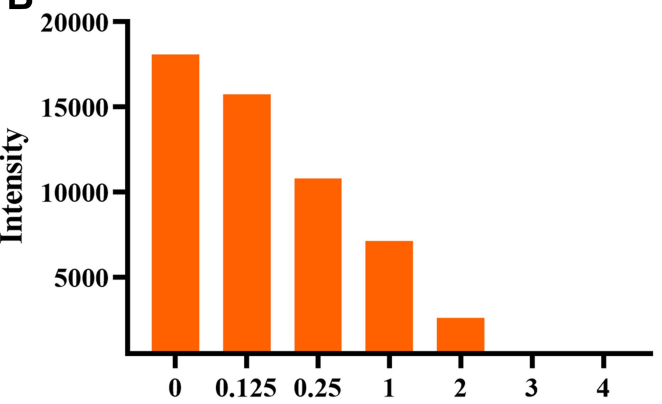

Concentration of SAL (ng/mL)

$\mathbf{F}$

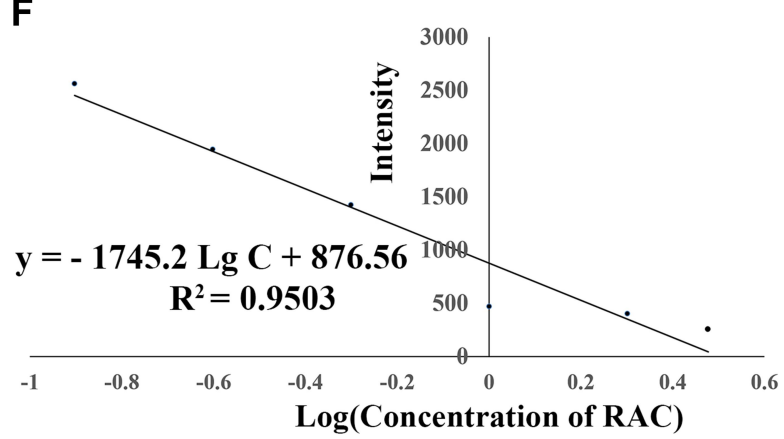

E

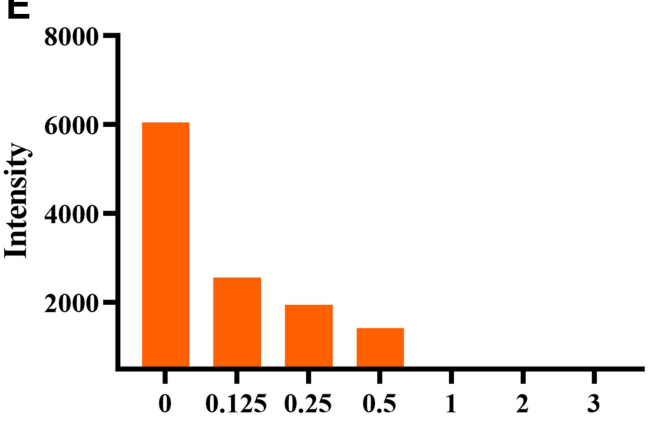

Concentration of RAC (ng/mL)

Figure 4 The sensitivity of the ractopamine (RAC) kit and salbutamol (SAL) kit. Detection results for salbutamol observed by the naked eyes (A), those constructed by GraphPad Prism (B) and fitting curve (C). Detection results for ractopamine observed by the naked eyes (E), those constructed by GraphPad Prism (F) and fitting curve (D). Orange boxes: result for sensitivity detection.

Abbreviations: $\mathrm{C}$, control line; $\mathrm{T}$, test line.

immunochromatographic strips to detect SAL and RAC in swine urine, the sensitivity of this test strip based on SeNPs was higher than the previously obtained $4 \mathrm{ng} / \mathrm{mL}$ (Supplementary Figure 2A and B) and $3 \mathrm{ng} / \mathrm{mL}$ (Supplementary Figure 2C and D) sensitivities.

We added clenbuterol, salbutamol, ractopamine, bambuterol and phenylethanolamine A to the Se-NP kit at 100 $\mathrm{ng} / \mathrm{mL}$ and observed the strips after 5 minutes (Figure 5). The kits had high specificity and no cross-reaction with other drugs. To estimate their stability, the Se-NP kits were stored at $40{ }^{\circ} \mathrm{C}$ for 30 days and then removed to examine the change in sensitivity. As shown in Figure 6, the SAL sensitivity was still $3 \mathrm{ng} / \mathrm{mL}$, and the RAC sensitivity was
$1 \mathrm{ng} / \mathrm{mL}$. The sensitivity of the test kits did not change, which indicates that the stability of the kits is good.

The Se-NPs used in this study offer excellent stability, sensitivity, and precision, and they are prepared by a simple method. The Se-NP kit we prepared is low in cost and has good specificity, stability and repeatability. It does not require other testing instruments, and the operation is convenient. The result is clear and easy to distinguish. This kit is therefore suitable for adapting to market site supervision and rapid detection. In this study, the $\mathrm{T}$ line of the SAL test strip was thicker than the $\mathrm{T}$ line of the RAC test strip because the antigen dilutions used in the two were different. In the later part of the study, it 

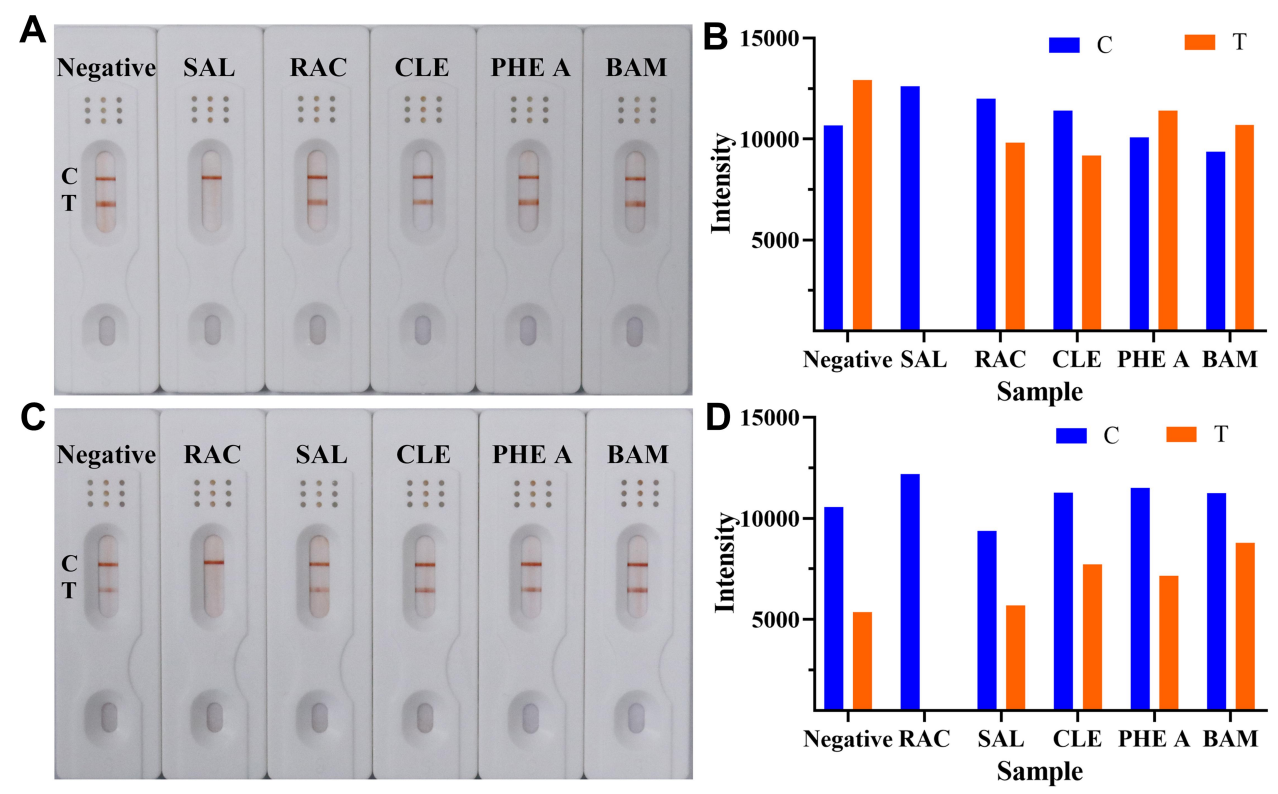

Figure 5 The specificity of the ractopamine (RAC) kit and the salbutamol (SAL) kit. Detection results for salbutamol observed by the naked eyes (A) and those constructed by GraphPad Prism (B). Detection results for ractopamine observed by the naked eyes (C) and those constructed by GraphPad Prism (D). Abbreviations: C, control line; T, test line; CLE, clenbuterol; BAM, bambuterol; PHE A, phenylethanolamine A.
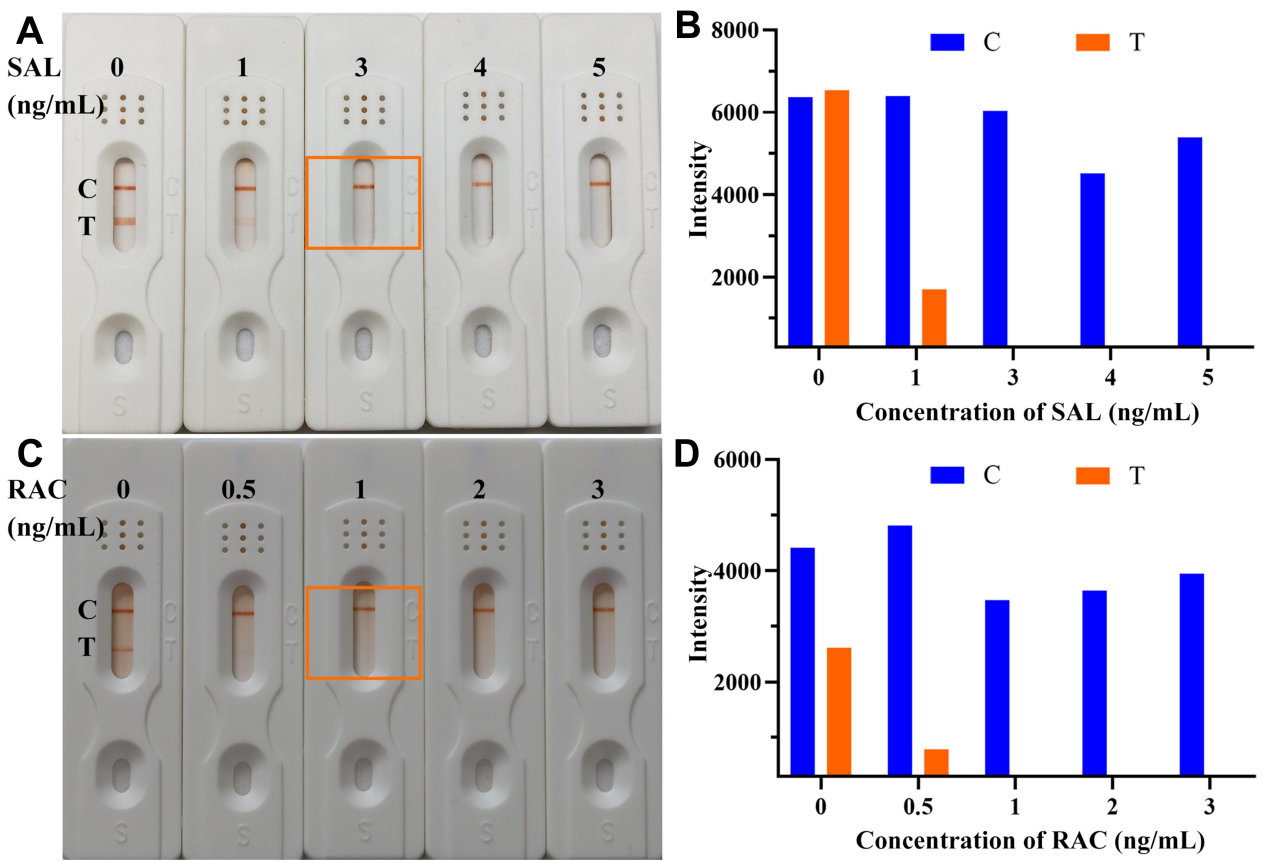

Figure 6 The stability of the ractopamine (RAC) kit and salbutamol (SAL) kit. Detection results for salbutamol observed by the naked eyes (A) and those constructed by GraphPad Prism (B). Detection results for ractopamine observed by the naked eyes (C) and those constructed by GraphPad Prism (D). Orange boxes: result for sensitivity of stability detection.

Abbreviations: C, control line; T, test line.

was found that the SAL T line often exhibited a hollow appearance. Sodium chloride is a strong electrolyte, which will affect the interaction between molecules. This may be the reason for the hollow band phenomenon.
To eliminate this phenomenon, the dilution component of the SAL antigen was changed. The RAC antigen dilution was PBS buffer, and the SAL antigen dilution was PB buffer. 
Table I Results of Recovery Experiment for RAC and SAL

\begin{tabular}{|c|c|c|c|c|c|c|c|}
\hline \multirow{2}{*}{\multicolumn{2}{|c|}{ Standard $(\mathrm{ng} / \mathrm{mL})$}} & \multicolumn{3}{|c|}{ Number of Experiment } & \multirow{3}{*}{$\begin{array}{c}\text { Ave } \\
0.5426\end{array}$} & \multirow[t]{2}{*}{ SD } & \multirow[t]{2}{*}{ CV\% } \\
\hline & & \multirow{2}{*}{$\frac{I}{0.5895}$} & \multirow{2}{*}{$\frac{2}{0.4890}$} & \multirow{2}{*}{$\begin{array}{c}3 \\
0.5494\end{array}$} & & & \\
\hline RAC & 0.5 & & & & & 0.0506 & $9.32 \%$ \\
\hline & 1.0 & 1.0728 & $\mathrm{I} .2034$ & 0.926 & $\mathrm{I} .0674$ & 0.1388 & $13.00 \%$ \\
\hline \multirow[t]{2}{*}{ SAL } & 0.5 & $0.4|8|$ & 0.5155 & 0.5367 & 0.4901 & 0.0632 & $12.91 \%$ \\
\hline & 1.0 & 1.1976 & 1.1272 & 0.9661 & 1.0970 & 0.1187 & $10.82 \%$ \\
\hline
\end{tabular}

\section{RAC and SAL Recovery Experiment}

For this purpose, $1 \mathrm{ng} / \mathrm{mL}$ and $0.5 \mathrm{ng} / \mathrm{mL}$ RAC standard was detected by the RAC ELISA kit, with three replicates for each concentration. A SAL recovery experiment was performed by the same method but with the SAL ELISA kit. The SD and CV\% values are shown in Table 1. The detection results of the recovery experiment are shown in Supplement Table 1 and calculated of RAC and SAL according to Supplementary Figure $3 \mathrm{~A}$ and $\mathrm{B}$, respectively.

\section{RAC and SAL Recovery Experiment}

A total of 16 samples were collected from a pig farm and were detected as negative for RAC and SAL by the corresponding ELISA kits, and the results are shown in $\underline{\text { Supplementary Tables } 2}$ and $\underline{3}$. The $\mathrm{C}$ line and $\mathrm{T}$ line were orange, indicating a negative result, regardless of whether the RAC kit or SAL kit based on Se-NPs was used. All of the results agreed well with those of the ELISA experiment (Figure 7). However, the number of collected samples was too small, and the sample size needs to be expanded for verification. In addition, collecting positive samples can better verify the efficacy of the kit.

\section{Discussion}

In the preparation process of the Se-NPs, the quality of water is very important. To obtain the ideal Se-NPs for the preparation of test strips, the ultrapure water from a Millipak terminal filter and Biopak terminal filter (Millipore) were compared. The results show that the Se-NPs prepared by the Millipak terminal filter were superior to those prepared by the Biopak terminal filter in terms of properties and colour

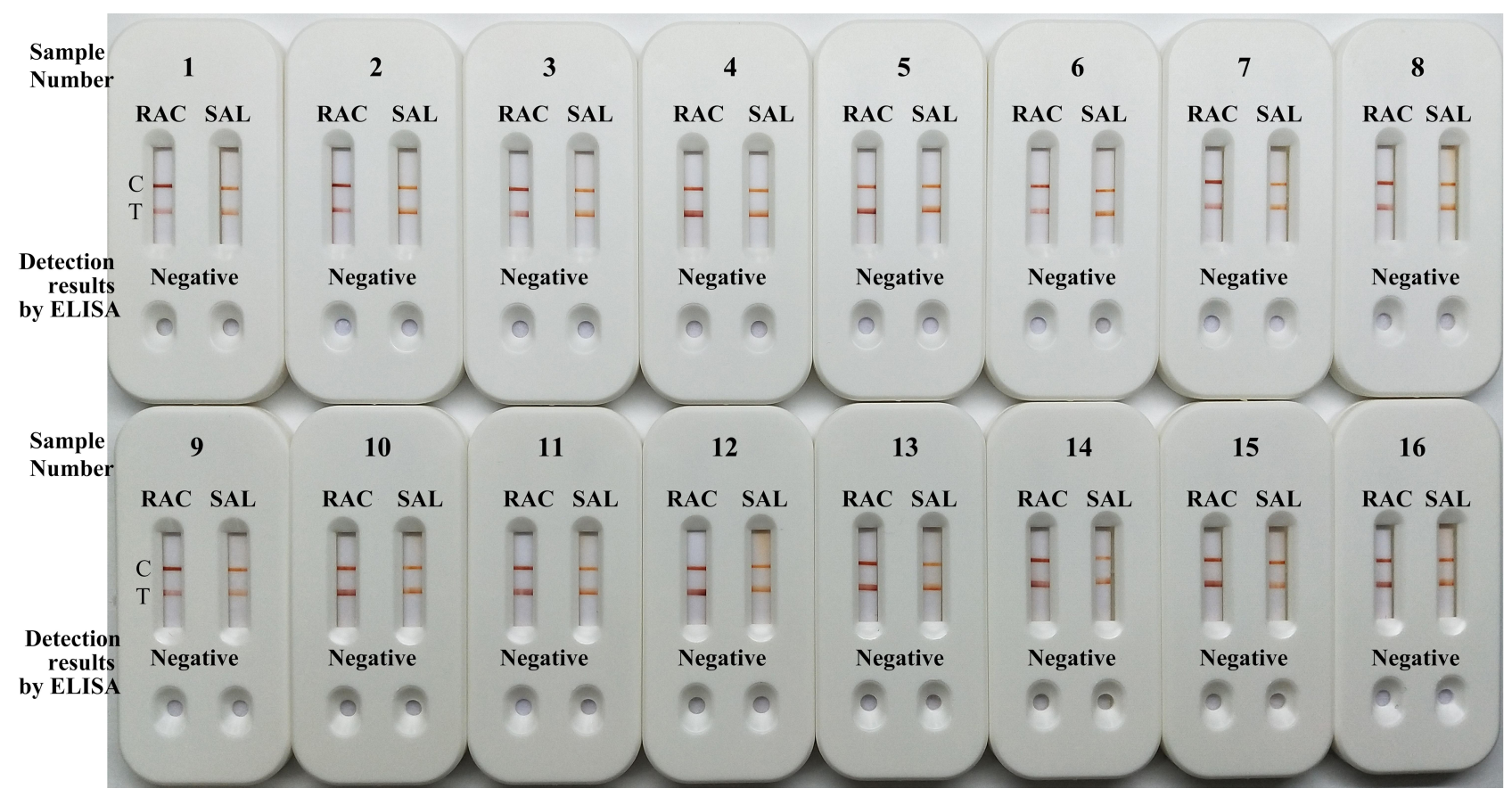

Figure 7 Detection results of actual samples using combined kits for salbutamol and ractopamine. Abbreviations: C, control line; T, test line; RAC, ractopamine; SAL, salbutamol. 
rendering. The water filtered through Millipak was bacteriafree and particle-free, and the water filtered through Biopak was pyrogen-free and nuclease-free. This suggests that bacteria and particles in water have a greater impact on the preparation of the Se-NPs. In summary, ultrapure water filtered through Millipak was selected for the preparation of the Se-NPs. The PDI value was less than 0.200, indicating that the distribution of the Se-NPs was uniform.

The Se-NPs maintains a stable state in water based on the presence of stabilizers, which is different from that of colloidal gold relying on the surface electric double layer to maintain a stable state in water. Colloidal gold conjugated with antibody or protein based on hydrogen bonding, hydrophobic interactions, van der Waals forces, etc. serves as evidence for the existence of the electric double layer. ${ }^{26}$ Whether the SeNPs also binds to proteins through these forces is not clear, which is what we are studying. The sizes of nanoparticles were measured by TEM and DLS. The SeNP size as determined by TEM was smaller than the DLS result, since the DLS measurement indicates the hydrated particle size and the TEM measurement determines the pure nanoparticle size. After antibody labelling on the surface of Se-NPs, the effect of water molecules may be eliminated, and the particle sizes obtained by TEM and DLS were similar.

The RAC concentration range of 16 swine urine samples was $0.0758 \mathrm{ng} / \mathrm{mL}-0.2584 \mathrm{ng} / \mathrm{mL}$, and the maximum value was lower than the sensitivity of the Se-NPs kit for RAC detection; the SAL concentration range of 16 swine urine samples was $0.0974 \mathrm{ng} / \mathrm{mL}$ $0.5281 \mathrm{ng} / \mathrm{mL}$, and the maximum value was lower than the sensitivity of the Se-NPs kit for SAL detection; these samples can thus be judged as negative samples for both RAC and SAL kits. One of the advantages of these kits is the naked eye interpretation, but the judgement results will be different due to the inconsistent colour discrimination ability of each person; therefore, each result was interpreted by three persons, and ImageJ software was used for auxiliary interpretation to ensure the accuracy of the results. The combined detection kit can present RAC and SAL test results, which offer greater advantages in terms of detection and cost. The combined detection kit was prepared by the following three methods: the conjugates of Se-NPs-anti-RAC $\mathrm{McAb}$ and Se-NPs-anti-SAL McAb were prepared in different tubes, and the above conjugates were sprayed into different pads for conjugate pad preparation; the conjugates of Se-NPs-anti-RAC McAb and Se-NPs-anti$\mathrm{SAL} \mathrm{McAb}$ were prepared separately, the above conjugate is sprayed into one pad for conjugate pad preparation; the conjugates of Se-NPs-anti-RAC McAb and Se-NPs-anti-SAL McAb were prepared in a tube, and the above conjugates were sprayed into one pad for conjugate pad preparation. The above methods were tried, but the RAC detection results were easily interfered by SAL, no matter if the RAC test line was on the side or between the control line and the SAL test line.

Swine urine composition will be affected by diseases such as hematuria, proteinuria and viral infections, ${ }^{27-29}$ but this composition change does not interfere with the performance of the RAC and SAL kit. RAC and SAL mainly exist in the form of original drugs in swine urine after being metabolized by the body, ${ }^{30}$ and metabolites will be produced at the same time. The metabolites of SAL identified in swine urine included glucuronide conjugate salbutamol, N-oxide-salbutamol, hydroxyl-salbutamol, methoxyl-salbutamol and dehydrated hydroxyl salbutamol, except SAL. ${ }^{31}$ The metabolites of RAC identified in swine was glucuronide conjugate ractopamine, except RAC. ${ }^{32}$ Whether the metabolite reacts with the corresponding kit requires further verification.

\section{Conclusions}

In this study, a rapid detection kit for the detection of RAC and SAL residues in pig urine was successfully developed by using immunochromatography with Se-NPs as a label. The sensitivities of the kits are $1 \mathrm{ng} / \mathrm{mL}$ for RAC and $3 \mathrm{ng} /$ $\mathrm{mL}$ for SAL. The results can be obtained within 5 minutes. The assay kit does not require any special device for reading the results, and the readout is a simple colour change that can be evaluated with the naked eye.

\section{Abbreviations}

Se-NPs, selenium nanoparticles; RAC, ractopamine; SAL, salbutamol; SDS, sodium dodecyl sulphate; PEG, polyethylene glycol; ELISA, enzyme-linked immunosorbent assay; HPLC, high-performance liquid chromatography; GC-MS, gas chromatography-mass spectrometry; LC-MS, liquid chromatography-mass spectrometry; CLIA, chemiluminescence immunoassay; ICA, immunochromatography; $\mathrm{H}_{2} \mathrm{SeO}_{3}$, selenous acid; $\mathrm{Ab}$, antibody; $\mathrm{McAb}$, monoclonal antibody; TEM, transmission electron microscope; DLS, dynamic light scattering; IgG, immunoglobulin $\mathrm{G} ; \mathrm{K}_{2} \mathrm{CO}_{3}$, potassium carbonate; PBS, phosphate-buffered saline; BSA, bovine serum albumin. 


\section{Acknowledgments}

We thank AJE for its linguistic assistance during the preparation of this manuscript.

\section{Author Contributions}

All authors made a significant contribution to the work reported, whether that is in the conception, study design, execution, acquisition of data, analysis and interpretation, or in all these areas; took part in drafting, revising or critically reviewing the article; gave final approval of the version to be published; have agreed on the journal to which the article has been submitted; and agree to be accountable for all aspects of the work.

\section{Funding}

This work was supported by the Science and Technology Department of Henan Province (grant no. 212102310174, 182102310154) and the National Natural Science Foundation of China (grant no. U1504823).

\section{Disclosure}

The authors declare that they have no competing interests.

\section{References}

1. Yan K, Zhang H, Hui W, et al. Rapid screening of toxic salbutamol, ractopamine, and clenbuterol in pork sample by high-performance liquid chromatography_UV method. J Food Drug Anal. 2016;24 (2):277-283. doi:10.1016/j.jfda.2015.12.002

2. Liang $\mathrm{X}$, Zhang $\mathrm{K}$, Zhang J, et al. Ractopamine residues in beef cattle hair during and after treatment. $J$ Anal Toxicol. 2016;40(2):153-158. doi:10.1093/jat/bkv135

3. Zhai F, Lai K, Zhang Y, Huang Y. Overview of $\beta$-doping residues in animal foods. Food Sci. 2011;32(5):351-356.

4. Yaeger MJ, Mullin K, Ensley SM, Ware WA, Slavin RE. Myocardial toxicity in a group of greyhounds administered ractopamine. Vet Pathol. 2012;49(3):569-573. doi:10.1177/0300985811424752

5. He P, Shen L, Liu R, et al. Direct detection of $\beta$-agonists by use of gold nanoparticle-based colorimetric assays. Anal Chem. 2011;83: 6988-6995. doi:10.1021/ac200769f

6. The Ministry of Agriculture and Rural Affairs of the People's Republic of China. Announcement No. 176 of the Ministry of Agriculture of the People's Republic of China; September 19, 2007. Available from: http://www.moa.gov.cn/ztzl/ncpzxzz/flfg/200709/t20070919_893060. htm. Accessed March 04, 2021

7. Tang Y, Gao J, Liu X, et al. Determination of ractopamine in pork using a magnetic molecularly imprinted polymer as adsorbent followed by HPLC. Food Chem. 2016;201:72-79. doi:10.1016/j. foodchem.2016.01.070

8. Zhang Q, Su Y, He Q, et al. Molecularly imprinted solid-phase extraction for the selective HPLC determination of ractopamine in pig urine. J Sep Sci. 2011;34(23):3399-3409. doi:10.1002/jssc.201100655

9. Jiang XF, Zhu YH, Liu XY. Identification of ractopamine glucuronides and determination of bioactive ractopamine residues and its metabolites in food animal urine by ELISA, LC-MS/MS and GC-MS. Food Addit Contam Part a Chem Anal Control Expo Risk Assess. 2014;31 (1):29-38. doi:10.1080/19440049.2013.855327
10. Karamolegou F, Dasenaki M, Belessi V, Georgakilas V, Thomaidis N. Multi-residue determination of $7 \beta$-agonists in liver and meat using gas chromatography-mass spectrometry. Food Anal Methods. 2018;11(10):2925-2942. doi:10.1007/s12161-018-1278-y

11. Wu J, Liu X, Peng Y. Determination of ractopamine in pig hair using liquid chromatography with tandem mass spectrometric detection. J Pharmacol Toxicol Methods. 2014;69(3):211-216. doi:10.1016/j. vasen.2014.02.001

12. Ma L, Nilghaz A, Choi JR, Liu X, Lu X. Rapid detection of clenbuterol in milk using microfluidic paper-based ELISA. Food Chem. 2018;246:437-441. doi:10.1016/j.foodchem.2017.12.022

13. Han S, Zhou T, Yin B, He P. Gold nanoparticle-based colorimetric ELISA for quantification of ractopamine. Microchim Acta. 2018;185 (4):210. doi:10.1007/s00604-018-2736-3

14. Zhao Y, Jiang D, Wu K, et al. Development of a sensitive monoclonal antibody-based ELISA for the determination of a $\beta$-adrenergic agonist brombuterol in swine meat, liver and feed samples. Anal Methods. 2016;8(38):6941-6948. doi:10.1039/C6AY01709F

15. Gao H, Han J, Yang S, Wang Z, Wang L, Fu Z. Highly sensitive multianalyte immunochromatographic test strip for rapid chemiluminescent detection of ractopamine and salbutamol. Anal Chim Acta. 2014;839:91-96. doi:10.1016/j.aca.2014.05.024

16. Liu R, Liu L, Song S, et al. Development of an immunochromatographic strip for the rapid detection of $10 \beta$-agonists based on an ultrasensitive monoclonal antibody. Food Agric Immunol. 2017;28 (4):625-638. doi:10.1080/09540105.2017.1309358

17. Zhang W, He X, Liu P, Li W, Liu X. Rapid determination of ractopamine in porcine urine by a fluorescence immunochromatography assay. Anal Lett. 2016;49(14):2165-2176. doi:10.1080/00032 719.2016.1138496

18. Hu LM, Luo K, Xia J, et al. Advantages of time-resolved fluorescent nanobeads compared with fluorescent submicrospheres, quantum dots, and colloidal gold as label in lateral flow assays for detection of ractopamine. Biosens Bioelectron. 2017;91:95-103. doi:10.1016/j. bios.2016.12.030

19. Liu H, Fang G, Wang S. Molecularly imprinted optosensing material based on hydrophobic CdSe quantum dots via a reverse microemulsion for specific recognition of ractopamine. Biosens Bioelectron. 2014;55:127-132. doi:10.1016/j.bios.2013.11.064

20. Liu D, Deng S, Lai W, Xia J. Development of lateral flow assay of ractopamine based on fluorescent microspheres. Food Machin. 2012;28(1):73-77.

21. Bai W, Huang H, Li Y, et al. Direct preparation of well-dispersed graphene/gold nanorod composites and their application in electrochemical sensors for determination of ractopamine. Electrochim Acta. 2014;117:322-328. doi:10.1016/j.electacta.2013.11.175

22. Wang Z, Jing J, Ren Y, et al. Preparation and application of selenium nanoparticles in a lateral flow immunoassay for clenbuterol detection. Mater Lett. 2019;234:212-215. doi:10.1016/j.matlet.2018.09.056

23. Wang Z, Zhi D, Zhao Y, et al. Lateral flow test strip based on colloidal selenium immunoassay for rapid detection of melamine in milk, milk powder, and animal feed. Int J Nanomed. 2014;9: 1699-1707. doi:10.2147/IJN.S58942

24. Wang $\mathrm{Z}$, Zheng $\mathrm{Z}$, $\mathrm{Hu} \mathrm{H}$, et al. A point-of-care selenium nanoparticle-based test for the combined detection of anti-SARSCoV-2 IgM and IgG in human serum and blood. Lab Chip. 2020;20:4255-4261. doi:10.1039/D0LC00828A

25. Li X, Zhang G, Deng R, et al. Development of rapid immunoassays for the detection of ractopamine in swine urine. Food Addit Contam Part a Chem Anal Control Expo Risk Assess. 2010;27(8):1096-1103. doi:10.1080/19440041003754985

26. Chandler J, Gurmin T, Robinson N. The place of gold in rapid tests. IVD Technol. 2000;6:37-49.

27. Tsai AT, Kuo CC, Kuo YC, et al. The urinary shedding of porcine teschovirus in endemic field situations. Vet Microbiol. 2016; 182:150-155. doi:10.1016/j.vetmic.2015.11.008 
28. Chade AR, Williams ML, Engel J, et al. A translational model of chronic kidney disease in swine. Am J Physiol Renal Physiol. 2018;315:F364-F373. doi:10.1152/ajprenal.00063.2018

29. Ghiggeri GM, Losurdo G, Ansaldi F, et al. Two cases of swine H1N1 influenza presenting with hematuria as prodrome. Pediatr Nephrol. 2010;25:779-780. doi:10.1007/s00467-009-1380-x

30. Smith DJ. The pharmacokinetics, metabolism, and tissue residues of - $\beta$-adrenergic agonists in livestock. J Anim Sci. 1998;76:173-194. doi: $10.2527 / 1998.761173 x$
31. Gu X, Liu YM, Yao T, et al. Identification of major metabolites of salbutamol in swine urine and plasma using ultra-high performance liquid chromatography-electrospray time of flight mass spectrometry. Chin J Anal Chem. 2014;42(11):1692-1696. doi:10.1016/S1872-204 0(14)60784-2

32. Qiang Z, ShenTu F, Wang B, et al. Residue depletion of ractopamine and its metabolites in swine tissues, urine, and serum. J Agric Food Chem. 2007;55(11):4319-4326. doi:10.1021/jf070167c

\section{Publish your work in this journal}

The International Journal of Nanomedicine is an international, peerreviewed journal focusing on the application of nanotechnology in diagnostics, therapeutics, and drug delivery systems throughout the biomedical field. This journal is indexed on PubMed Central, MedLine, CAS, SciSearch ${ }^{\mathbb{R}}$, Current Contents ${ }^{\mathbb{R}} /$ Clinical Medicine, $^{-}$
Journal Citation Reports/Science Edition, EMBase, Scopus and the Elsevier Bibliographic databases. The manuscript management system is completely online and includes a very quick and fair peer-review system, which is all easy to use. Visit http://www.dovepress.com/ testimonials.php to read real quotes from published authors. 